\title{
Capacity of MIMO Channels: a Volumetric Approach
}

\author{
L. W. Hanlen and M. Fu \\ School of Electrical Engineering and Computer Science \\ The University of Newcastle, N.S.W. 2308 Australia
}

\begin{abstract}
This paper deals with the problem of computing channel capacity for wireless communications systems with multi-antennas. The system is modelled using a transmit volume, a receive volume and a set of reflective scatterers. Based on the authors' previous work on channel modelling of such a system, we give a multi-input-multi-output model for communications between arbitrary antenna arrays in two volumes. Together with a novel noise model for describing interferences, we derive formulas for channel capacity and capacity bounds. In particular, our results give conclusions which agree with intuition: In the case where the transmitter knows the channel, the channel capacity saturates to a constant when the number of transmit/receive antennas increases beyond a certain threshold; In the case where the transmitter does not know the channel, the channel capacity still saturates when the receive antennas increases but decays when the number of transmit antennas increases beyond a certain threshold.
\end{abstract}

\section{INTRODUCTION}

There has been a rush of research work in the area of multi-antenna wireless communications in the last few years. Much of it was motivated by the work of [1], [2] which shows the potential significant increase in channel capacity by using multi-antennas for both transmission and receiving. The work of [1], [2] is done under similar assumptions that 1) the multi-input-multi-output (MIMO) channel model has independent components with complex Gaussian (or Rayleigh) distributions; 2) the noises at different receive antennas are independent and white; and 3) the channel is known to the receiver but not to the transmitter. Their main result is that the channel capacity of such a system grows linearly with respect to the minimum number of transmit or receive antennas. This is known as the linear growth property.

Since [1], [2], some studies have been done on channel modelling of multi-antenna systems. Several authors [3], [4] have investigated the validity of the so-called "rich" scattering environment, which is required to achieve the linear growth. The work of [5] addressed the case where the receiver elements are placed closely within a fixed region of space, and proposed a limit to capacity by placing an artificial restraint

A part of this work was carried out while the authors visited Nanyang Technological University in Singapore. The authors would like to thank Nanyang Technological University for their support to this research. on the received signal power. Likewise, other authors [6], [7] have suggested that a correlation of the signals at the receivers will occur, as the element separation becomes "small."

Recently, we have proposed a volumetric approach to study the problem of channel modelling for wireless communications systems involving a transmit volume, a receive volume, and a set of reflective scatterers [8]. Based on the work of [8], we give a MIMO model for communications between arbitrary antenna arrays in two volumes. Together with a novel noise model for describing interferences, we have derived formulas for channel capacity and capacity bounds. Our main interest is to study the capacity limit when the numbers of transmit and receive antennas in the two volumes increase. Two cases have been studied: 1) The channel is known to the transmitter; and 2) The channel is unknown to the transmitter. In the former case, we show that the channel capacity saturates to a constant when the number of transmit/receive antennas increases beyond a certain threshold. In the latter case, the channel capacity still saturates when the receive antennas increases but decays when the number of transmit antennas increases beyond a certain threshold.

The contributions of the paper are as follows: 1) We provide a numerical approach for computing the channel capacity and capacity bound for multi-antenna systems with scattering. 2) We give a novel noise model which describes interference noises in the receive volume based on communications modes. In comparison with the common assumption of independent noises in receive antennas, our model better represents interference noises in the receive volume. More precisely, we do not have the unrealistic consequence (as the independent noise assumption would) that the received signalto-noise ratio can be increased indefinitely by placing more and more receive antennas. Also, no artificial scaling (as in [5]) is needed on the receive antennas. 3) The results can be used as a guideline for determining the numbers and spacing of transmit and receive antennas in a given communication environment.

This paper is arranged as follows: Section II gives a review of the channel modelling approach in [8]. In section III, we provide a multi-antenna channel model together with a noise model. Section IV gives channel capacity formulas and 


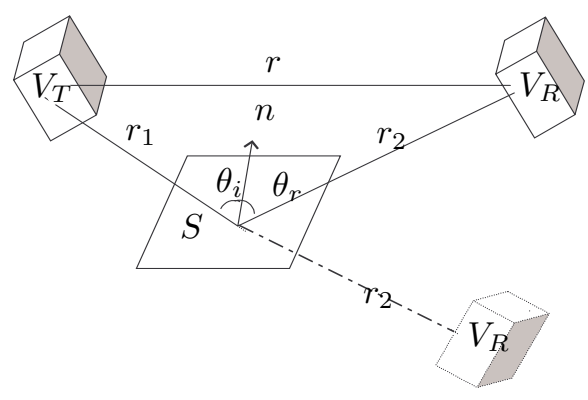

Fig. 1. Multi-antenna Communications System

capacity bounds. A simulation example is given in section $\mathrm{V}$ and some conclusions are drawn in section VI.

\section{Channel Model}

Consider two arbitrary volumes $V_{T}$ and $V_{R}$ separated by a distance $r$ along the $z$ direction, as shown in figure 1. In addition, there are scatterers in the space which are assumed to be pure reflectors. Only one such scatterer is shown in figure 1. Volume $V_{T}$ contains a source wave function ${ }^{1} \psi\left(\mathbf{r}_{T}\right)$ which generates a wave function $\phi\left(\mathbf{r}_{R}\right)$ in the volume $V_{R}$ for points $\mathbf{r}_{T}$ in $V_{T}$ and $\mathbf{r}_{R}$ in $V_{R}$. It is assumed that the source signal is monochromatic (or narrowband).

A set of $N_{T}$ transmitters are placed at locations $\rho_{T n}, n=$ $1,2, \ldots N_{T}$ in $V_{T}$. Similarly, $N_{R}$ receivers are placed at $\rho_{R n}, \quad n=1,2, \ldots N_{R}$ in $V_{R}$. In this section, we shall ignore the antennas and concentrate on the communications modelling between the two volumes. This allows us to avoid the limitations placed by the numbers and locations of the antennas. We will return to the antennas in section III.

The work presented in this section is summarized from [8] which is in turn motivated by [9]. The paper [9] studied the problem of channel modelling between two volumes without scatterers for optical communications. In [8], we generalized the work in [9] and provided a numerical algorithm for computing the channel model for wireless communications between two volumes with or without scatterers.

Given a source wave function $\psi\left(\mathbf{r}_{T}\right)$ in the transmit volume $V_{T}$, the wave function generated by this source function at any spatial location $\mathbf{r}=(x, y, z)$ is given by

$$
\phi(\mathbf{r})=\int_{V_{T}} G\left(\mathbf{r}, \mathbf{r}_{T}\right) \psi\left(\mathbf{r}_{T}\right) d^{3} \mathbf{r}_{T}
$$

where the function $G\left(\mathbf{r}, \mathbf{r}_{T}\right)$ is the free-space Green's function given by

$$
G\left(\mathbf{r}, \mathbf{r}_{T}\right)=\frac{\exp \left(-\iota k\left|\mathbf{r}-\mathbf{r}_{T}\right|\right)}{4 \pi\left|\mathbf{r}-\mathbf{r}_{T}\right|}
$$

\footnotetext{
${ }^{1}$ Wave functions may be considered as the continuous equivalent of pointsources - in fact we may simply write point-sources as special examples of wave functions.
}

and $k=2 \pi / \lambda$ is the wave number for a wavelength $\lambda$ and $\iota=\sqrt{-1}$.

Each scatterer is assumed to be a pure reflective plane of sufficient size that we may neglect fringing [8]. When "hitting" a scatterer, the signal $\phi(\mathbf{r})$ is reflected with a possible attenuation coefficient $\rho$ and then continues to travel in space. Therefore, the transmitter is effectively also transmitting to a mirror-imaged copy of $V_{R}$ (as shown in figure 1, provided the angle of the reflector is appropriate. Consequently, $V_{T}$ and $V_{R}$ may communicate via different surfaces through different scatterers, thus increasing the number of communications modes. In addition, the connection strength of a particular communications mode can be increased if this mode is shared by several paths.

However, one can quickly conclude from the above that there is a limit to the number of communications modes and their connection strengths because the amount of space available for scattering is limited. Our work in [8] gives a method for computing the channel model for a given system setting, as described below.

The direct path can be modelled as

$$
\phi\left(\mathbf{r}_{R}\right)=\int_{V_{T}} G\left(\mathbf{r}_{R}, \mathbf{r}_{T}\right) \psi\left(\mathbf{r}_{T}\right) d^{3} V_{T}
$$

where $\phi\left(\mathbf{r}_{R}\right)$ denotes the wave function at location $\mathbf{r}_{R} \in$ $V_{R}$ by $\psi\left(\mathbf{r}_{T}\right)$ through the direct path. Following the ideas in [9], two sets of orthonormal basis functions, $\left\{\beta_{T i}\left(\mathbf{r}_{T}\right)\right\}$ and $\left\{\beta_{T j}\left(\mathbf{r}_{T}\right)\right\}$ can be obtained to describe the signal transfer between $V_{T}$ and $V_{R}$. That is, if we decompose any $\psi\left(\mathbf{r}_{T}\right)$ and $\phi\left(\mathbf{r}_{R}\right)$ as

$$
\psi\left(\mathbf{r}_{T}\right)=\sum_{i} a_{i} \beta_{T i}\left(\mathbf{r}_{T}\right)
$$

and

$$
\phi\left(\mathbf{r}_{R}\right)=\sum_{j} b_{j} \beta_{R j}\left(\mathbf{r}_{R}\right)
$$

respectively, then the coefficient vectors $a$ and $b$ are related by a transfer matrix $\Gamma$ as follows:

$$
b=\Gamma a
$$

where the $(j, i)$ th element of $\Gamma$ is given by

$$
\gamma_{j i}=\int_{V_{R}} \int_{V_{T}} \beta_{R j}^{*}\left(\mathbf{r}_{R}\right) G\left(\mathbf{r}_{R}, \mathbf{r}_{T}\right) \beta_{T i}\left(\mathbf{r}_{T}\right) d^{3} \mathbf{r}_{T} d^{3} \mathbf{r}_{R}
$$

Moreover, if the basis functions are chosen to be the eigenfunctions of (3), $\Gamma$ becomes a diagonal matrix (i.e., we have a diagonal model) and $\gamma_{i i}$ are simply the singular values of the transfer matrix. The number of communications modes corresponds to the number of significant singular values.

A similar approach can be applied to each scattering path through the use of the "mirror-imaged" communications path, and a transfer matrix is obtained. This transfer matrix is in 
general a non-diagonal matrix. A singular value decomposition may be applied to obtain a diagonal transfer matrix which is then truncated to an appropriate size by removing insignificant singular values. The remaining size corresponds to the number of communications modes for the overall system.

For notational simplicity, we will still denote the orthonormal basis functions for $V_{T}$ and $V_{R}$ for the overall system by $\beta_{T i}\left(\mathbf{r}_{T}\right)$ and $\beta_{R j}\left(\mathbf{r}_{R}\right)$, respectively. The number of communications modes will be denoted by $N_{c}$. The overall channel transfer matrix will still be denoted by $\Gamma$ and it is a diagonal matrix with elements $\gamma_{i}, i=1, \ldots, N_{c}$. We note that $\gamma_{i}$ are roughly the same for most $i$ and they drop down quickly when $i$ reaches $N_{c}$.

See [8] for the details of the channel modelling process and a numerical algorithm for computing the channel model.

\section{Multi-Antenna Modelling}

In this section, we consider the modelling problem with transmit and receive antennas. Suppose we place $N_{T}$ transmitters at arbitrary locations $\rho_{T n}, n=1,2, \ldots N_{T}$, in $V_{T}$. Similarly, $N_{R}$ receivers are placed at arbitrary locations $\rho_{R n}, n=1,2, \ldots N_{R}$, in $V_{R}$. We may denote the vector of transmit signals as $x$ and the vector of received signals as $y$. Our aim is to develop the channel model for the system:

$$
y=H x+w
$$

where $w$ represents the receiver noise and $H$ is the transfer matrix between $x$ and $y$. The remainder of this section will show how $H$ and $w$ may be modelled.

\section{A. Receiver Modelling}

We assume the receivers are ideal. That is, each receiver $k$ samples the wave function $\phi\left(\mathbf{r}_{R}\right)$ at a location $\rho_{R k}$. We may write the received signal $y$ as:

$$
y_{k}=\phi\left(\rho_{R k}\right)+w_{k}
$$

Using (5), we get:

$$
y=B b+w
$$

where

$$
B=\left[\begin{array}{ccc}
\beta_{R 1}\left(\rho_{R 1}\right) & \cdots & \beta_{R N_{c}}\left(\rho_{R 1}\right) \\
\vdots & & \vdots \\
\beta_{R 1}\left(\rho_{R N_{R}}\right) & \cdots & \beta_{R N_{c}}\left(\rho_{R N_{R}}\right)
\end{array}\right]
$$

\section{B. Noise Modelling}

We propose to model the noise function in $V_{R}$ as follows:

$$
\phi_{w}\left(\mathbf{r}_{R}\right)=\sum_{j=1}^{N_{c}} \eta_{j} \beta_{R_{j}}\left(\mathbf{r}_{R}\right)
$$

where $\eta_{j}$ is an independent white noise with zero mean and variance $\sigma_{j}^{2}$. That is, the noise of the channel excites the eigen-modes of the receiver. So that the noise in $V_{R}$ should be independent of the receive antennas, and receivers sample signal and noise. This corresponds to the practical situation where the main noise source comes from interferences in the environment rather than from the receivers themselves. If we have fewer receivers than modes, the noise samples will appear independent.

Only the noise components in the subspace of $\left\{\beta_{R_{j}}\left(\mathbf{r}_{R}\right)\right\}_{j=1}^{N_{c}}$ are considered because other noise components can be removed by the receivers via projection (provided enough receive antennas are there to sample the noises). Hence, when sampled by the receive antennas, we get

$$
w=B \eta
$$

\section{Transmitter modelling}

We shall assume that the transmitters are ideal point sources of small volume. Each transmitter is able to produce a signal $x_{n}$ independent of any other transmitters. Therefore, we may choose the source function $\psi\left(\mathbf{r}_{T}\right)$ as:

$$
\begin{aligned}
\psi\left(\mathbf{r}_{T}\right) & =\sum_{n=1}^{N_{T}} \psi_{n}\left(\mathbf{r}_{T}\right) \\
\psi_{n}\left(\mathbf{r}_{T}\right) & = \begin{cases}\frac{1}{\sqrt{\Delta V_{T}}} x_{n} & \mathbf{r}_{T} \in V_{T n} \\
0 & \text { otherwise }\end{cases}
\end{aligned}
$$

where $x_{n}$ is the intensity of source $n, \Delta V_{T n}$ is the (small) volume occupied by source $n$ and $\Delta V_{T}$ is the size of the volume. For simplicity, it is assumed that all volumes are of equal size, but this can be relaxed. The scaling of $1 / \sqrt{\Delta V_{T}}$ in (14) is used so that the transmission power for transmitter $n$ is $\left|x_{n}\right|^{2}$ and is independent of $\Delta V_{T}$. If $\Delta V_{T}$ is sufficiently small, the projection of $\psi_{n}\left(\mathbf{r}_{T}\right)$ onto $\beta_{T}\left(\mathbf{r}_{T}\right)$ may be simplified as:

$$
\begin{aligned}
a_{n i} & =\int_{V_{T}} \psi_{n}\left(\mathbf{r}_{T}\right) \beta_{T i}\left(\mathbf{r}_{T}\right) d^{3} \mathbf{r}_{T} \\
& =\sqrt{\Delta V_{T}} x_{n} \beta_{T i}\left(\rho_{T n}\right)
\end{aligned}
$$

We then write $a_{i}$ as the sum of $a_{n i}$ :

$$
a_{i}=\sqrt{\Delta V_{T}} \sum_{n=1}^{N_{T}} x_{n} \beta_{T i}\left(\rho_{T n}\right)
$$

In vector form we have:

$$
a=\sqrt{\Delta V_{T}} A x
$$

where the $N_{c} \times N_{T}$ matrix $A$ is given by:

$$
A=\left[\begin{array}{ccc}
\beta_{T 1}^{*}\left(\rho_{T 1}\right) & \cdots & \beta_{T 1}^{*}\left(\rho_{T N_{T}}\right) \\
\vdots & & \vdots \\
\beta_{T N_{c}}^{*}\left(\rho_{T 1}\right) & \cdots & \beta_{T N_{c}}^{*}\left(\rho_{T N_{T}}\right)
\end{array}\right]
$$


Combining the modelling results for the receivers and transmitters, the channel may be written as:

$$
y=\sqrt{\Delta V_{T}} B \Gamma A x+B \eta
$$

\section{CAPACITY}

In this section, we proceed to estimate the channel capacity using the model (19). We are interested in the scenario where the numbers of transmit and receive antennas are both large. Two cases are analyzed: 1) The channel model is known to the transmitter; 2) The channel model is unknown to the transmitter. In both cases, the receiver knows the channel model (e.g., through channel estimation).

\section{A. Channel known at transmitter}

We consider the case $N_{T} \gg N_{c}$ and $N_{R} \gg N_{c}$. If $N_{R}$ is sufficiently large, (i.e., the sampling points $\rho_{R n}$ are sufficiently dense), we may approximate $B^{*} B$ by an integral as:

$$
\left[B^{*} B\right]_{i, j} \approx \frac{N_{R}}{V_{R}} \int_{V_{R}} \beta_{R i}^{*}\left(\mathbf{r}_{R}\right) \beta_{R j}\left(\mathbf{r}_{R}\right) d \mathbf{r}_{R}^{3}
$$

Since the basis $\beta_{R}\left(\mathbf{r}_{T}\right)$ is orthonormal, from (11) we write:

$$
B^{*} B \approx \frac{N_{R}}{V_{R}} I_{N_{c}}
$$

In similar fashion we may write:

$$
A A^{*} \approx \frac{N_{T}}{V_{T}} I_{N_{c}}
$$

for $N_{T}$ sufficiently large. Define

$$
\hat{y}=\frac{V_{R}}{N_{R}} B^{*} y \quad x=\sqrt{\frac{V_{T}}{N_{T}}} A^{*} \hat{x} \quad \hat{\eta}=\frac{V_{R}}{N_{R}} B^{*} B \eta
$$

and re-write (19) as:

$$
\hat{y}=\sqrt{\Delta V_{T}} \frac{V_{R}}{N_{R}} B^{*} B \Gamma \sqrt{\frac{V_{T}}{N_{T}}} A A^{*} \hat{x}+\hat{\eta}
$$

Using (21) and (22), we may simplify (24) as:

$$
\hat{y}=\sqrt{\frac{\Delta V_{T} N_{T}}{V_{T}}} \Gamma \hat{x}+\hat{\eta}
$$

Further, it is easy to verify that the $\eta_{i}$ are approximately independent with variance $\hat{\sigma}_{i}^{2}=\sigma_{i}^{2}$.

Recall that the channel transfer matrix $\Gamma$ is diagonal and the singular values $\gamma_{i}$ are roughly equal. Hence, the optimal transmission scheme is equal power per communication channel:

$$
\mathcal{E}\left\{\hat{x} \hat{x}^{*}\right\}=\frac{P}{N_{c}} \cdot I_{N_{c}}
$$

Hence, the channel capacity of the system is given by

$$
C \approx \sum_{i=1}^{N_{c}} \log _{2}\left(1+\frac{\hat{P}_{i}}{\hat{\sigma}_{i}^{2}}\right)
$$

where $\hat{P}_{i}$ is the signal power in $\hat{y}_{i}$, given by

$$
\hat{P}_{i}=\frac{\Delta V_{T} N_{T}}{V_{T}} \frac{P}{N_{c}} \gamma_{i}^{2}
$$

Simplifying the above, we get

$$
C \approx \sum_{i=1}^{N_{c}} \log _{2}\left(1+\frac{\Delta V_{T} N_{T}}{V_{T}} \frac{\gamma_{i}^{2}}{N_{c}} \frac{P}{\sigma_{i}^{2}}\right)
$$

Since $\Delta V_{T} N_{T} \leq V_{T}$, we further have

$$
C \lesssim \sum_{i=1}^{N_{c}} \log _{2}\left(1+\frac{\gamma_{i}^{2}}{N_{c}} \frac{P}{\sigma_{i}^{2}}\right)
$$

which gives the upper bound on the channel capacity. In particular, the bound is independent of $N_{T}$ and $N_{R}$, provided that they are sufficiently large.

Note that the assumption that $\gamma_{i}$ are roughly the same can removed. In this case, the power distribution among different diagonal channels should be done using the waterfilling principle.

\section{B. Channel unknown at transmitter}

When the channel is unknown at the transmitter, the optimal transmit strategy is to assign independent equal-power signals to all transmitters, i.e.,

$$
\mathcal{E}\left\{x x^{*}\right\}=\frac{P}{N_{T}} I_{N_{T}}
$$

Using (19) and (23), we get

$$
\hat{y} \approx \sqrt{\Delta V_{T}} \Gamma A x+\hat{\eta}
$$

where $\hat{\eta}$ is the same as before.

For simplicity, we assume $\sigma_{i}=\sigma$ (constant). The channel capacity is thus given by

$$
\begin{aligned}
C & \approx \log _{2} \operatorname{det}\left(I+\frac{\Delta V_{T} \Gamma A A^{*} \Gamma P / N_{T}}{\sigma^{2}}\right) \\
& \approx \log _{2} \operatorname{det}\left(I+\frac{\Delta V_{T} N_{T}}{V_{T}} \frac{\Gamma^{2} P / N_{T}}{\sigma^{2}}\right) \\
& =\sum_{i=1}^{N_{c}} \log _{2}\left(1+\frac{\Delta V_{T}}{V_{T}} \frac{P \gamma_{i}^{2}}{\sigma^{2}}\right)
\end{aligned}
$$

Hence, the conclusion is that the channel capacity decreases as $\Delta V_{T}$ decreases (or $N_{T}$ increases), provided $N_{T}$ is sufficiently large. The reason for this is subtle:

All communication between $V_{T}$ and $V_{R}$ occurs through the connecting eigen-modes. The (point-source) transmit elements excite the finite transmit modes. If we have densely placed transmitting elements, all of which are transmitting white signals, the transmit modes will become saturated. From a beamforming perspective we would say "the receiver cannot distinguish between the sources." At this point additional transmitters act as interference sources. 


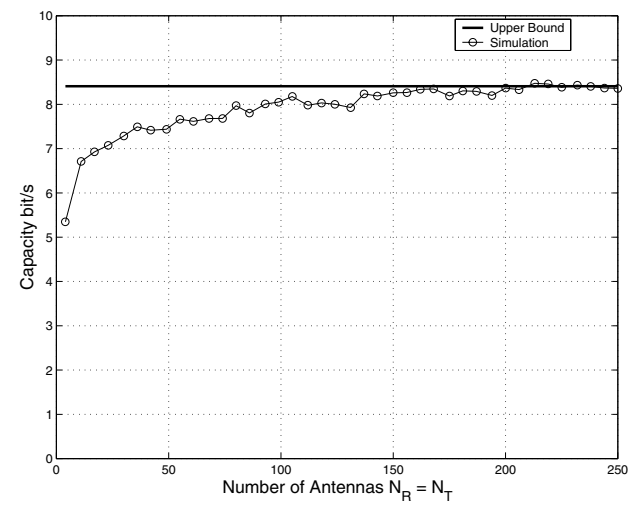

Fig. 2. Capacity vs $N_{T}=N_{R}$ for direct transmission channel (known at transmitter) for $N_{c}=2.1$

The capacity bound is independent of $N_{R}$ because no new information on either the signal or noise can be gained by placing more receive antennas, provided $N_{R}$ is already sufficiently large.

\section{Simulation}

The capacity bounds provided in the previous section assume $N_{T}$ and $N_{R}$ are large. In this section, we provide a simulation example to examine how the capacity changes when $N_{T}$ and $N_{R}$ change.

In figure 2 we have simulated a direct transmission channel (without scattering). The volumes $V_{T}$ and $V_{R}$ were two parallel prisms whose sizes are $15 \lambda \times 9 \lambda \times 9 \lambda$ and $18 \lambda \times 9 \lambda \times 9 \lambda$, respectively. The direction of propagation is along the last ( $z$ ) axis. The volumes are separated by a distance $r=100 \lambda$, giving the total number of communication modes $N_{c}=2.1$. The position of each antenna element was chosen uniformly at random from within the respective volume. Basis functions were generated using [8], and interpolated for each position.

For the purpose of the simulation, we set $N_{T}=N_{R}$ and $\Delta V_{T} \cdot N_{T}=V_{T}$. The transmission power and noise was normalized as follows:

$$
\frac{P}{(4 \pi \mathbf{r})^{2} \sigma^{2}}=10
$$

The channel was assumed to be known to the transmitters. The largest singular value of the equivalent continuous channel was given by $\nu_{1}=0.0059$. A water-filling method was used by the transmitter in both the upper bound and the simulation. This avoided the need for the equal gains assumption.

The solid line shows the capacity bound as given by (29), whereas the circled line shows how the capacity increases as $N_{T}$ (and $N_{R}$ ) increases. It can be seen that the capacity asymptotes below the bound given by (29) as predicted.

\section{CONCLUSIONS}

In this paper, we have analyzed the channel capacity for multi-antenna communications between two volumes with or without scattering bodies. Bounds are given to the channel capacity for two cases: channel known to the transmitter, and channel unknown to the transmitter. The main findings of the paper are that in the known channel case, the channel capacity is bounded as the number of antennas increases, whereas in the unknown channel case, the channel capacity actually decreases as the number of transmit antennas increases beyond a certain value.

In this paper, the channel model is extended to a model between transmit and receive antenna arrays. Further, a novel noise model is provided to properly characterize the receiver noises. The results of this paper should not be interpreted as a discouragement for using multi-antennas. Our capacity bounds simply indicate that there is a limit to the number of antennas one can place in a fixed volume (for either transmission or receiving). In fact, the channel capacity is limited by the transmit and receive volumes and scatterers. To increase the capacity, one needs to increase the available transmit/receive volumes, hence allowing more antennas to be placed. The results of this paper can be used to give a guideline for determining the numbers of antennas necessary to utilize the available capacity.

\section{REFERENCES}

[1] I. Telatar, "Capacity of multi-antenna gaussian channels," European Transactions on Telecommunications, vol. 10, no. 6, pp. 585-595, Nov. 1999.

[2] G. Foschini and M. Gans, "On limits of wireless communications in a fading environment when using multiple antennas," Wireless Personal Communications, vol. 6, pp. 311-335, 1998.

[3] D. Chizhik, G. Foschini, M. Gans, and R. Valenzuela, "Keyholes, correlations, and capacities of multielement transmit and receive antennas," IEEE Trans. Commun., vol. 1, no. 2, pp. 361-392, Apr. 2002.

[4] D. Gesbert, H. Bölcskei, D. Gore, and A. Paulraj, "MIMO wireless channels: Capacity and performance prediction," in IEEE Global Telecommunications Conference, Globecom'00, vol. 2, 2000, pp. 1083 1088.

[5] N. Chiurtu, B. Rimoldi, and E. Telatar, "Dense multiple antenna systems," in IEEE Information Theory Workshop, Cairns, Australia, Sept. 2-7 2001, pp. 108-109.

[6] T. Abhayapala, R. Kennedy, and J. Ho, "On capacity of multi-antenna wireless channels: Effects of antenna separation and spatial correlation," in Proc. 3rd Australian Communications Theory Workshop (AusCTW), 2002, pp. 100-104.

[7] A. Abdi and M. Kaveh, "A space-time correlation model for multielement antenna systems in mobile fading environments," IEEE J. Select. Areas Commun., vol. 20, no. 3, pp. 550-560, Apr. 2002.

[8] L. Hanlen and M. Fu, "Wireless communications with spatial diversity: A volumetric approach," in IEEE International Conference on Communications, ICC'03, Anchorage, Alaska, May. 11-15 2003, to appear.

[9] D. Miller, "Communicating with waves between volumes: evaluating orthogonal spatial channels and limits on coupling strengths," Applied Optics, vol. 39, no. 11, pp. 1681-1699, Apr. 2000. 